\title{
Desde la crisis de la pregunta: Prácticas y reflexiones en torno a los trabajos finales de graduación en la formación de docentes
}

\author{
From the Crisis of Questioning: Considerations About Practices and Reflections on \\ Graduation Projects
}

\section{A partir da crise da pergunta: práticas e reflexões ao redor dos trabalhos finais de graduação na formação de docentes}

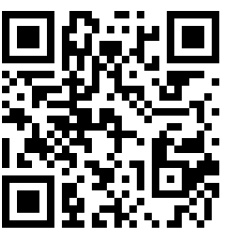

iD

ceciliadobles347@gmail.com

Cecilia Dobles-Trejos

Universidad Nacional

Heredia, Costa Rica

cid.org/0000-0003-2027-6613

Patricia Ramírez-Abrahams

Universidad Nacional

Heredia, Costa Rica

pramirezabrahams@gmail.com

https://orcid.org/0000-0002-3958-6008

Ana lsabel Herrera-Castro

Universidad Nacional

Heredia, Costa Rica

aherre0@gmail.com

https://orcid.org/0000-0002-6073-4378

Recibido • Received • Recebido: 28 / 09 / 2018

Corregido • Revised • Revisado: 07 / 12 / 2019

Aceptado • Accepted • Aprovado: 31 / 03 / 2020

Resumen: Este artículo parte de la experiencia de un grupo de personal académico de la División de Educación Básica, del Centro de Investigación y Docencia en Educación, de la Universidad Nacional, Costa Rica, que conforman la Comisión de Trabajos Finales de Graduación de esa unidad académica. El objetivo de este artículo es plantear una visión retrospectiva y reflexiva sobre la formación universitaria de profesionales en Pedagogía y el aporte de la investigación en dicha formación, un enfoque dialógico para contribuir a promover el desarrollo personal (personal docente-personal investigador) crítica, reflexivamente y con capacidad de asombro en su trabajo. Esto se hace desde los retos y desafíos que propone un quehacer pedagógico fundamentado en la pregunta de 
http://doi.org/10.15359/ree.24-2.24

ROR: https://ror.org/01t466c14 Universidad Nacional, Costa Rica

http://www.una.ac.cr/educare

educare@una.cr

investigación, como forma de construcción del conocimiento sobre sus propias prácticas docentes. El enfoque de una investigación en la formación universitaria con sentido y propósito (Mora Alvarado y Gómez Torres, 2017) que parte de la reflexión en torno a la pregunta como base de la relación dialógica de la praxis pedagógica, se constituye en el sustento teórico de este ensayo. Se plantea la investigación como proceso, deja atrás la visión tradicional de que una propuesta de investigación parte de un "anteproyecto" y no como un proceso en que el estudiantado y su docente de tutoría caminan juntos para avanzar en trayectorias de producción de conocimiento que permitan el crecimiento profesional y personal de ambos sujetos. En otras palabras, se trata de no ver el trabajo final de graduación como un fin en sí mismo, sino como un vehículo que provee herramientas al estudiantado para desarrollar las destrezas de investigación como medio y como experiencia para recuperar la capacidad de asombro, sobre todo para saberse capaz de construir nuevo conocimiento.

Palabras claves: Capacidad de asombro; la pregunta de investigación, formación docente; investigación; trabajo final de graduación.

Abstract: This article is based on the experience of some male and female academics from the Basic Education Division, Center for Research and Teaching in Education of Universidad Nacional (the Graduation Project Faculty Committee). It offers a conversational approach aimed at promoting personal growth for both researchers and faculty members. It does so based on a retrospective view of university training of educators, with support from research in that field, in a critical, reflexive way, and involving their capacity for astonishment. Besides, it considers the challenges posed by pedagogical tasks based on the research question as a way to make discoveries about their teaching practices. This research focus on university formation with meaning and purpose (Mora Alvarado and Gómez Torres, 2017) starts from a reflection on questions as a basis for a dialogical relationship in the pedagogical practice and is the theoretical support for this essay. Research is seen as a process, leaving behind the traditional view that a research proposal starts from a "blueprint." Instead, it unites students and tutors, working together to bring about knowledge to grow both personally and professionally. In other words, it is not about thinking of graduation projects as ends in and of themselves, but rather as a way to provide students with tools to expand research skills as a means to recover their capacity for astonishment, and especially to acknowledge their ability to create new knowledge.

Keywords: Capacity for astonishment; graduation project; the research question, teaching training; research.

Resumo: Este artigo está baseado na experiência de um grupo de acadêmicos e acadêmicas da Divisão da Educação Básica, do Centro de Investigação e Docência em Educação, da Universidade Nacional, que formam a Comissão de Trabalhos Finais de Graduação dessa unidade acadêmica. Nele se expõe, desde uma visão retrospectiva sobre a formação universitária do profissional em Pedagogia e a colaboração da investigação em dita formação, uma abordagem dialógica que visa contribuir e promover o desenvolvimento pessoal (docente-pesquisador) crítico, reflexivo e com a capacidade de assombro no seu trabalho. Isso se faz desde as metas e desafios que propõe uma tarefa pedagógica fundamentada na questão da pesquisa, como forma de construção do conhecimento sobre suas próprias práticas docentes. Este é um tipo de investigação na formação universitária com sentido e propósito (Mora Alvaraqdo e Gómez Torres, 2017), a partir da reflexão em torno a pergunta como base da relação dialógica da prática pedagógica, se constitui no sustento teórico desse artigo. 
Coloca-se a pesquisa como um processo, deixando para trás a visão tradicional de que uma proposta de investigação parte de um "anteprojeto" e não como um processo em que tanto estudantes como docentes caminham juntos para avançar num processo de produção de conhecimento que permita o crescimento profissional e pessoal de ambos. Em outras palavras, trata-se de não ver o trabalho final de graduação como um fim em si mesmo, senão como um veículo que provê ferramentas ao estudante para desenvolver as destrezas da pesquisa como meio e como experiência para recuperar a capacidade de assombro, e sobretudo ser capaz de construir um novo conhecimento.

Palavras chaves: Capacidade de assombro; a pergunta de investigação, formação docente, pesquisa; trabalho final de graduação.

\section{Introducción}

En la División de Educación Básica del Centro de Investigación y Docencia en Educación de la Universidad Nacional, Costa Rica, se imparten las carreras de Pedagogía en cuatro diferentes énfasis: Educación Preescolar, I y II Ciclos, Educación Especial y Enseñanza del Inglés para I y II Ciclos. Todos los planes de estudio de las carreras mencionadas plantean, en su propuesta de formación docente, la investigación como un eje que permea los procesos educativos universitarios. En el caso de Preescolar, I y II Ciclos y Educación Especial, culminan con la realización de un trabajo final de graduación, conducente al grado de licenciatura.

La instancia encargada de la aprobación y seguimiento de las propuestas del estudiantado es la Comisión de Trabajos Finales de Graduación (CTFG). En la última década, esta comisión ha liderado procesos de reflexión y análisis sobre la investigación en el campo de estudio de la pedagogía, promoviendo la reflexión hacia una visión integral, crítica, constructiva y humanista de la investigación en el quehacer pedagógico.

La práctica universitaria en estas carreras ha llevado a que en los cursos de investigación que se ofrecen en el nivel de licenciatura se elaboren los anteproyectos de los trabajos finales de graduación. En consecuencia, la construcción de dichos anteproyectos ha tenido diferentes momentos; antes del 2008, en la División de Educación Básica (DEB) durante el primer semestre el estudiantado profundizaba en sus conocimientos de investigación, y en el segundo semestre iniciaban la elaboración de su anteproyecto, el cual se presentaba a la CTFG una vez que se concluían los cursos del nivel de licenciatura.

Surge entonces una reflexión, a partir del año 2008, sobre el impacto de esta práctica curricular estipulada en los diferentes planes de estudio citados. Este momento de pensamiento fue liderado por el personal académico que integró la CTFG de la DEB ese año', quienes

\footnotetext{
${ }^{1}$ Académicos y académicas que conformaban la CTFG en el 2008: Dr. Rafael Esteban Jiménez, Dra. Susana Ruiz,
} M.Sc. Cecilia Dobles T. 
propusieron hacer una revisión de la propuesta que estaba vigente en ese momento. Se tomó en cuenta una serie de aspectos que incidían en la visión y papel de la investigación en la formación de docentes de este país, con miras a las nuevas necesidades y exigencias del futuro para profesionales de educación en un contexto nacional que, desde décadas atrás, viene discutiendo sobre la crisis en su propuesta educativa. Según el Estado de la Educación (Programa Estado de la Nación, 2008):

La débil presencia de la tríada teoría-investigación-práctica en la mayor parte de los planes de formación es un vacío clave que debe superarse, dada su gran importancia para el desarrollo de educadores autónomos y críticos, capaces de actualizarse y adaptarse a contextos diversos. (p. 124)

El Programa Estado de la Nación (2008) menciona experiencias internacionales analizadas en materia de formación docente, y se rescata que un aspecto que puede contribuir a una formación docente pertinente en los tiempos actuales es:

El desarrollo de capacidades de investigación-reflexión-acción que sirvan para vincular la teoría con la práctica, de tal manera que esta esté presente a lo largo de toda la carrera, y cultivo, en los alumnos, de una disposición a la reflexión, la rendición de cuentas, la resolución de problemas y el aprendizaje permanente. (p. 36)

Lo anterior apela a la revisión del papel de los distintos actores y actoras en el escenario educativo, y dentro de estos, las universidades que forman a maestros y maestras.

A partir de ese año 2008, se facilita un proceso en el cual el estudiantado aprende a "investigar investigando", es decir, es mediante las preguntas que se plantean, como parte del diseño de su TFG, desde los cursos de investigación, que el estudiantado se aproxima al conocimiento de la teoría del método y la investigación. Los cursos de investigación se reformulan para que las investigaciones, propuestas mediante anteproyectos, surjan de las necesidades de los contextos en los que se desarrollará la investigación de manera que, desde el curso de Investigación I, el estudiantado de licenciatura plantee su propuesta de investigación. A esta se le dará continuidad en el curso de Investigación II, donde se consolida su trabajo de campo y profundización en la formación de sus destrezas investigativas, tomando como base el desarrollo de las primeras fases de su TFG, ya acompañado en este momento, además, por un profesor o profesora de tutoría.

Este cambio se fundamenta en un profundo posicionamiento epistemológico en relación con cómo se asume la realidad investigada, la persona investigadora frente a esa realidad y en cómo se religan esas interacciones en la construcción del conocimiento mutuo. De ahí que 
la complejidad de las realidades en las que se investiga se nutre a partir de la formulación de diferentes preguntas que surgen de la cotidianidad en la que se investiga y de las reflexiones que se promueven desde una formación universitaria que llega a culminar con la elaboración del TFG.

\section{Desde la crisis de la pregunta ... una aproximación conceptual a la investigación como proceso}

El abordaje del problema de investigación desde la academia universitaria tiene posturas epistemológicas definidas, sin embargo, algunos de estos encuadres teóricos, metodológicos y ontológicos no necesariamente están planteados para la investigación en pedagogía, es decir, no reconocen esa naturaleza formativa y educadora necesaria en un proceso investigativo que se desarrolla en la diversidad de los procesos educativos (Mora Alvarado y Gómez Torres, 2017).

Cuando vemos llegar a las aulas universitarias estudiantes que por doce o más años han sido formados en una propuesta educativa en la que no hay un diálogo creativo, sino más bien una aceptación ciega y silenciosa de los conocimientos que tengo y debo recibir, todo para cumplir con una meta de "escolarización" que socialmente tiene sus valores y en torno a la cual se construye un mito fundacional de la identidad costarricense: somos un pueblo educado.

Esa lógica aplastante se refleja en un discurso nacional y prácticas tradicionales en las aulas que, a su vez, revocan y reducen el ejercicio pedagógico a una propuesta unidireccional y vertical. En esta el estudiantado escasamente preguntará y mucho menos será capaz, a través del cuestionar su diario vivir, de construir conjuntamente con sus propios estudiantes propuestas asertivas para su desarrollo integral y contribuir a la sociedad. Esa premisa ha sido ampliamente explicada por Freire y Faundez (2013):

Es esto que llamo "castración de la curiosidad". Lo que está sucediendo es un movimiento unilineal que va de aquí para allá y punto, no hay regreso, y ni siquiera hay una demanda jel educador de manera general, ya trae la respuesta sin que se le haya preguntado algo! (p. 69)

Esto nos lleva a plantear una cuestión fundamental para generar cambios importantes en los procesos de aprendizaje a este estudiantado, que viene del sistema educativo y ahora son universitarios y universitarias: ¿cuáles herramientas podemos ofrecerles para desaprender todos esos conocimientos que marcaron un camino limitado para la posibilidad de construir conocimiento desde una mirada creativa, crítica y reflexiva?

Estamos hablando de maestros y maestras para el futuro que, de no romper este "círculo vicioso" de prácticas de transmisión de conocimiento pasivo y acrítico, en las que no están presentes la inquietud intelectual y la reflexión pertinente y contextualizada, cuando sean profesionales llevarán la misma propuesta a las aulas. De ahí que veamos la investigación en 
la formación docente como un reto que les permitirá adquirir, o mejor dicho "redescubrir", la capacidad del asombro y la pregunta ante las demandas pedagógicas que les propone la vivencia cotidiana.

Las licenciaturas de las carreras de la DEB de la Universidad Nacional se han propuesto, desde el año 2008, hacer una revisión exhaustiva de esta situación, y construir una propuesta que ofrezca al estudiantado universitario herramientas para derribar sus temores y reconocer sus destrezas, para que de una manera reflexiva y crítica pueda proponer y ser parte de su ejercicio pedagógico en conjunto con sus estudiantes.

La propuesta se plantea desde los cursos de investigación, partiendo de que el estudiantado pueda iniciar un proceso que le facilite asumir el coprotagonismo de una "aventura" de construcción de conocimiento en diálogo con los sujetos y realidades con las que trabajarán. A su vez, este ejercicio reflexivo y crítico procura las bases para su formación en investigación. El eje guía de este proceso es propiciar que los grupos de estudiantes recobren su capacidad de asombro y de cuestionamiento de la realidad para proponer cambios, mediante propuestas dialógicas que les permitan construir nuevo conocimiento, tanto como estudiantado universitario como con las personas con quienes van a trabajar para realizar su TFG.

Lo anterior desde las implicaciones de una postura de docente, en que los procesos de aprendizaje, en el contexto universitario, están permeados por el desarrollo de la capacidad reflexiva que se podría producir desde la reflexión de las acciones que se promueven en las relaciones teórico prácticas del conocimiento, las cuales se construyen mediante la investigación en relación con la pedagogía. Así lo señala Jiménez (2012):

La exploración del conocimiento práctico supone recurrir a otras nociones claves como la de reflexión. Muestra de ello, diversos trabajos tratan de discernir entre constructos teóricos con el objetivo de clarificar los procesos implicados en el pensamiento práctico del profesorado. Estudios como el de Gholami y Husu (2010) plantean diferencias entre constructos como conocimiento practicable y conocimiento praxial, o aportaciones como la de Hagger y Hazel (2006) sobre el procedimiento de teorización práctica. Concretamente, la teorización práctica constituye un proceso donde investigación y formación se fusionan, donde la reflexión y la acción se interrelacionan. A partir de este proceso se valida el pensamiento práctico, que va mucho más allá, de la mera reproducción de tradiciones docentes. En ambos casos, se pone el acento en la necesaria reflexión del docente sobre su propia práctica, la revisión de sus formas de actuar y la valoración de las experiencias educativas más relevantes. (Jiménez, 2012, p. 241)

Heidegger y Gadamer (mencionados por Rillo et ál., 2011), plantean la necesidad del diálogo desde una postura dialéctica, para propiciar un reconocimiento y construcción mutua entre sí y 
con el entorno, para lo cual la pregunta-respuesta se convierte en el ente dinamizador y el eje que permite una mayor profundización en la lectura y comprensión de la realidad. Esta realidad, asumida desde una visión formativa, requiere un abordaje pedagógico diferente en el aula universitaria, en particular en la concepción que se tiene de la investigación en la formación docente.

Estos temas han sido discutidos desde diversas posturas, que cuestionan si el ejercicio pedagógico actual es capaz realmente de permitir al estudiantado universitario desarrollar posturas reflexivas desde la comprensión básica de su papel en la sociedad, aspecto en el que, si queremos exhaustividad, deberíamos remontarnos a los Diálogos de Platón, base de la mayéutica. Es decir, si quien educa en la actualidad es capaz de permitir un ejercicio dialógico con sus estudiantes, a través de la pregunta, a fin de posibilitar la construcción conjunta de conocimiento, coconstruyendo y proponiendo las herramientas para que en el futuro puedan construir sus propios caminos, con miras a poner la simiente para tener una sociedad más justa. Al respeto Rillo et ál. (2011) plantean:

Se abre una posibilidad para establecer el diálogo con aquél que no tiene voz, de manera que la lógica de la pregunta y la respuesta adquiere un horizonte práxico que permite la comprensión e interpretación del mundo de la vida en la que se encuentra situado el ser humano. (p. 4)

Freire (1979) plantea la importancia del diálogo, desde la "palabra" que se debe reconocer en la praxis, que toma forma en tanto hay una persona que reconoce el peso de sus palabras y el impacto que estas pueden tener en la realidad. En la palabra, intención y conciencia van de la mano, pues consideran respetuosamente a la persona con quien se interactúa, sus necesidades, propuestas y preguntas, lo que se constituye en la base fundante de una propuesta de la pedagogía dialógica. Esa propuesta considera la pregunta como eje que moviliza ese diálogo, ya que es necesario reconocer desde dónde la otra persona construye su visión de la realidad, cuáles son las preguntas que se hace sobre las propuestas que está planteando y cómo, a partir de esas preguntas, empieza a construir su propio conocimiento.

Es importante recordar el concepto de "investigación" como construcción de un nuevo conocimiento. ¿Pero cómo construyo ese nuevo conocimiento, sobre todo cuando se trata de la práctica pedagógica? Lo construyo desde mi propia "visión de lo que debe ser", o lo construyo en diálogo con las otras personas, para asegurar que haya una reflexión-crítica de sus vivencias y, a su vez, una apropiación de los nuevos conocimientos que estamos construyendo desde esa praxis.

Lo anterior nos lleva a reflexionar que la manera en cómo ha cambiado la pregunta tiene que ver con el cambio que tiene el estudiantado universitario con respecto a su papel como profesional en la realidad educativa nacional. Por ello, es importante promover esta visualización en la persona que ingresa a una carrera universitaria, a fin de que asuma la pedagogía como 
una ciencia dialógica que se construye desde el conocimiento en la acción y la reflexión, con el fundamento de su práctica en un escenario sociocultural complejo y diverso, que toma como base la pregunta para construir conjuntamente con los educandos nuevo conocimiento.

Si bien se reconoce, mediante el diálogo, la posición de las otras personas con las que interactúo para construir nuevo conocimiento, es importante subrayar que el autorreconocimiento es fundamental. Es decir, desde dónde me aproximo a las otras personas con las que voy a interactuar en una propuesta de investigación-pedagógica. Por tanto:

La formación de profesores y profesoras [que] ha de sustentarse en teorías que se ocupen de la extensión de la conciencia del ser humano como ser cultural y social (Kincheloe, 2001 , p. 41). Por tanto, ha de transitar, necesariamente, por el reconocimiento del modo en que las opiniones políticas, creencias religiosas, roles y representaciones de género, el concepto de uno mismo/a, los valores o las perspectivas educativas, reciben el influjo de la cultura. Así como también, percibir el carácter de las situaciones concretas, el momento actual que vive la persona, el estado emocional, las tensiones del contexto y las circunstancias que lo envuelven, y cómo esto incide en las propias conductas.

Son escasas las perspectivas formativas que desembocan en un desarrollo profesional holístico. Es decir, que incorpore dimensiones culturales e identitarias, emocionales, cognitivas y conductuales, redundando, especialmente, en una enseñanza íntegra, sensible a la identidad, al ámbito personal, a las emociones y que no resulte sólo técnica. (Korthagen, 2010, citado por Jiménez, 2012, p. 240)

La claridad en la posición que tenga ante la realidad, la conciencia en cómo interactúo y por qué interactúo de tal o cual manera, me lleva a reconocer de dónde vienen mis preguntas sobre la realidad, por qué me asombro y por qué esa pregunta o asombro son válidos en un proceso investigativo. Esta seguridad sobre mis propias ideas me da la base también para reconocer que, si entro en un proceso dialógico con las otras personas, mis preguntas se irán transformando y replanteando conforme conozco y nos conocemos.

\section{Bases fundantes de la identidad del pedagogo o la pedagoga y su incidencia en su participación como sujeto investigador}

La visión de la investigación dentro de la Unidad Académica a la que pertenecen las carreras mencionadas anteriormente es clara en los planes de estudio, pero nuevamente volvemos a esa disyuntiva en la que los planes nos proponen una idea y la realidad nos empuja por los caminos sobre los cuales sentimos más inseguridad, asumiendo que nuestro estudiantado también así lo siente. En la DEB, la investigación es un eje transversal en la malla curricular; sin embargo, la 
práctica se desarrolla sobre todo en área de investigación y apunta a que se revisa como una materia más que debemos aprender. Estaba ubicada, hasta el 2017, en tres momentos de la formación del estudiantado universitario: un curso en diplomado, un curso bachillerato y dos en licenciatura.

Visto desde fuera, esta es una buena propuesta, pues se asume que la transversalización se apoyaría en estos cursos, pero no entregaría todo el peso del quehacer investigativo en la formación pedagógica sobre ellos, pues los demás cursos de la malla lo deberían articular. Sin embargo, la necesidad de cubrir contenidos y acercarse a una realidad únicamente como sujetos observadores no participantes da muy poco margen para poder desarrollar la destreza de preguntar y preguntarle a la realidad, y mucho menos de asumir una postura críticapropositiva-dialógica ante aquello que observo.

Esta situación es compleja, pues no solo se puede reducir a una práctica universitaria. También se debe contemplar la visión social e histórica de lo que debe ser un maestro o una maestra y la propuesta educativa que requiere un país inmerso en la lógica del mercado en pleno siglo XXI, entre otros aspectos a tomar en cuenta.

El punto es cómo, a partir de la propuesta curricular y metodológica que estamos desarrollando en los cursos de investigación en licenciatura, se puede contribuir a incorporar la investigación en la formación de una manera transversal. En ese sentido, apuntamos a que el proceso que se realiza actualmente brinde un espacio para que el estudiantado, mediante un diálogo con la realidad que le interesa conocer, pueda conocerse a sí mismo, en todas las posibilidades (humanas y profesionales).

Históricamente se ha discutido la posición de un sujeto docente investigador, pero no se ha considerado si la investigación que se produce en la práctica pedagógica cumple o no con los cánones propuesto por la academia. Esta situación ha repercutido en reconocer que la investigación sea parte de la identidad del quehacer pedagógico. En las décadas de 1970 y 1980 la discusión fue asumida por distintos estudios, entre ellos destaca el de Stenhouse (2003), quien plantea la importancia del sujetodocente investigador: "El ideal es que la especificación del [currículo] aliente una investigación y un programa de desarrollo personales por parte del profesor, mediante el cual éste aumente progresivamente la comprensión de su propia labor y perfeccione así su enseñanza" (p. 195). Esas propuestas, que se discutían ya en esas décadas, señalaban el papel docente circunscrito al aula escolar o colegial.

En el contexto anteriormente mencionado, es importante recordar la vieja discusión entre la validez de la teoría versus la práctica, en términos de la supremacía que históricamente se le ha dado a la construcción teórica producto del quehacer "científico", con lo que se negaba toda posibilidad de construir nuevo conocimiento válido desde la práctica. 
En esa línea Kemmis, 1999), coincide en lo siguiente:

No sólo ... tenemos que interesarnos tanto por las teorías de los "prácticos" como lo estamos por sus prácticas, y que debemos estudiar las prácticas de los "teóricos" de forma tan minuciosa como lo hacemos con sus teorías, sino que demuestra también que el trabajo de la práctica educativa y el de la elaboración teórica deben desarrollarse conjuntamente. (p. 19)

La práctica educativa ha sido vista en relación de subordinación, no solo en la posibilidad de producir conocimiento, sino también ante las propuestas inamovibles del sistema educativo, que define los derroteros sobre cuáles deben guiar el proceso de enseñanza-aprendizaje, llevando esta situación a la homologación de la práctica a una actividad o activismo en el ejercicio docente.

Esto tiene consecuencias serias, que aún hoy han sido difíciles de vencer. La práctica pedagógica se investiga desde fuera por tradición, pues no se da el reconocimiento para que docentes puedan investigar su propia práctica y proponer acciones claras a partir de sus hallazgos. Es el otro sujeto, el que sabe, el que determina si "mi práctica es correcta", con lo que se pone en tela de juicio la posibilidad de construir conocimiento desde el ejercicio práctico del quehacer pedagógico.

La pedagogía se "realiza en la práctica", pero requiere de la"pregunta"; de lo contrario, ¿qué es lo que orienta el quehacer pedagógico? Al quitar la pregunta que se genera en la reflexión sobre la acción, se elimina la posibilidad de crear con el otro ser, lo convertimos en un ejercicio técnico. Esto, claramente entendido, desde el valor de la reflexión sobre el propio actuar como camino para mejorar el quehacer docente.

No se trata de formar a personas investigadoras solamente desde el reconocimiento del "conocimiento verdaderoy medible", sino de docentes que investigan desdela capacidad deasombro, de preguntar para convertir su práctica pedagógica en un ejercicio crítico-propositivo-dialógico, que permita proponer el disfrute de la construcción de nuevo conocimiento conjuntamente con sus estudiantes: que sea productor de una experiencia vital de auto y mutuo conocimiento.

Si no damos estas herramientas en la formación universitaria, ponemos en una posición de desventaja a los futuros grupos de profesionales de la educación, pues fácilmente los sueños de realizar cambios y coincidir con una mejor calidad de la educación son captados por el modelo de funcionamiento del sistema educativo. Es así como esa ruptura entre teoría y práctica, en lo cotidiano, se hace evidente cuando en las aulas escolares solo se reproduce lo solicitado por el sistema educativo.

En esa relación dialéctica entre teoría y práctica es bueno escudriñar sobre los hilos que permiten un quehacer crítico en el ejercicio pedagógico. En ese sentido, es fundamental 
reconocer la propia visión que se tiene de las personas con las que interactúa y construye el hecho pedagógico, personas que están en un contexto histórico y que tienen una visión y caminar propio en el proceso de enseñanza y aprendizaje.

Esavisiónseentrelazaconotroaspectotambiénmuyimportante,yeselautorreconocimiento de cada docente en este ejercicio, hacia dónde quiero marchar, qué impacto tiene mi ejercicio profesional, y si ese ejercicio profesional se vincula con el sueño de país o de mundo que tengo.

Lo anterior no podría darse sin la vinculación a un tercer aspecto fundamental en esta propuesta: mi mirada hacia el futuro, desde esa mirada que construyo en el presente, y desde ahí el cuestionamiento fundamental de cómo me estoy acercando a las otras personas en el ejercicio pedagógico, la ética con la que defino la manera en que conozco y construyo nuevo conocimiento conjuntamente con las personas con quienes interactúo cotidianamente.

Freire (1997) propone en su libro Pedagogía de la autonomía. Saberes necesarios para la práctica educativa, que "enseñar exige investigación. No hay enseñanza sin investigación ni investigación sin enseñanza" (p. 31). Es evidente que esta temática estaba puesta sobre las mesas de discusión, dentro y fuera de las academias, desde la relación intrínseca y simbiótica de la pedagogía y la investigación como procesos que se nutren y permiten el acercamiento profundo a la complejidad del escenario donde se desarrollan.

El punto es que esa investigación que se realiza en las aulas, como parte del ejercicio profesional pedagógico, muchas veces en la teoría y sobre todo en la práctica no se considera investigación. Al respecto, Patterson y Shannon (2002) mencionan que "aparentemente los maestros tienen la convicción de que la mayor parte de la investigación en educación es prácticamente irrelevante; esta podría ser una consecuencia directa de los estándares de rigor en la investigación científica". (p. 25). Es indudable que esa postura ha incidido en la identidad del profesorado y su interés y convicción en reconocer la investigación como parte de su quehacer profesional.

En este punto es necesario enfatizar en la diferencia de lo que plantean los textos sobre el concepto de pedagogía y la reflexión que buscamos en este documento: desentrañar la incidencia de la manera en que los futuros grupos profesionales construyen su identidad, entendiéndola como la plantea Cirese (citado por Giménez,1996) como: "un reconocerse en ... algo que tal vez sólo en parte coincide con lo que efectivamente uno es" (p.187). Esto nos lleva a enfatizar el carácter de construcción social que adquiere la definición de mi identidad y la identidad nuestra como un grupo social específico. Efectivamente, nuestra identidad no es solo lo que yo quiero ser, sino lo que las condiciones sociales, políticas, culturales y económicas han contribuido en su conformación.

Visto así, pesa en esa construcción de identidad colectiva una mirada hacia una pedagogía que la invalida como un área que produce conocimiento, pues está vinculada a una práctica donde la función educativa, tradicional e históricamente, se ha entendido como la de transferir 
información, propiciar que "el otro lo tenga". Visto así, el maestro o maestra se limita a mediar entre el que produce información y quien la recibe, y no un sujeto investigador de su propio ejercicio pedagógico y constructor de conocimiento, que se recrea y crea la propuesta pedagógica cada vez que está departiendo con sus estudiantes.

Esa mirada ha calado en la autodefinición del pedagogo o la pedagoga que, ciertamente, se sustenta en una práctica que está legitimada por propuestas educativas enmarcadas en lógicas regionales y nacionales, en la realización de una práctica cotidiana en los centros educativos y en la afirmación de esta identidad desde las aulas universitarias que, muchas veces, por olvido o por omisión, preparan profesionales para que cumplan dichas funciones. Giménez (1996) coincide con lo anterior y afirma:

Por eso la identidad no es un atributo o una propiedad intrínseca del sujeto, sino que tiene un carácter intersubjetivo y relacional. Esto significa que resulta de un proceso social, en el sentido de que surge y se desarrolla en la interacción cotidiana con los otros. El individuo se reconoce a sí mismo sólo reconociéndose en el otro. (p. 188)

La identidad no es una autoadscripción, es producto de un proceso intersubjetivo en el que todo contribuye a la reafirmación de "cómo me defino". En ese sentido, para el caso docente hay que tomar en cuenta la práctica en los centros educativos, las políticas nacionales que definen sus funciones, la propuesta de formación en las universidades, la autodefinición en función de los parámetros que la sociedad espera del personal docente, entre otros aspectos importantes que inciden, incluso, en la percepción que se tiene sobre sí y el desempeño profesional.

Es interesante ver en retrospectiva, como ya Freire lo planteaba, la necesidad de abordar la relación docente-dicente de manera dialéctica, donde el fin era la producción de conocimiento, proceso en el cual ambos seres se constituían en sujetos de un proceso educativo. Sus planteamientos, desde una postura latinoamericana, están urgidos de un reconocimiento del sujeto como eje del proceso de construcción de conocimiento y no como mero objeto, tanto si lo vemos desde el estudiante como desde el docente. En ese sentido, una discusión similar se estaba dando en Europa en la última mitad del siglo pasado, cuando se hablaba de la necesidad de la investigación del objeto, en tanto que esta era planteada por un sujeto que definió qué y cómo quería investigar. En América Latina podemos reconocer las propuestas de Maturana y Francisco Varela, con la autopoiesis, cuando se refieren a "sistemas circulares, recursivos, autorreferentes y paradójicos" (Matura y Varela, 1999, citados por Ibáñez, 1994, p. xv).

Aquí nos toca reconocer nuestra responsabilidad en esta situación, esto es, con cuáles parámetros estamos fortaleciendo la identidad de los futuros grupos profesionales. En consecuencia, surge otra situación importante de abordar: la contradicción entre lo que decimos y cómo lo ponemos en práctica. En los planes de estudio de las carreras de la DEB se señala la 
necesidad de formar un estudiantado crítico y propositivo; sin embargo, las prácticas que la mayoría de cursos propone se circunscriben a realizar un ejercicio que se propone desde el aula universitaria, en el cual se debe responder a un objetivo que la temática propuesta requiera.

Durante esas prácticas, las posibilidades de asombrarse son limitadas, pues se requiere tomar la información que el espacio de tiempo dentro de un aula permita. En esa condición, la posibilidad de profundizar con una mirada sobre la complejidad es limitada, pero sobre todo más limitada la capacidad de reconocerme, de saber cuál es mi postura ante la realidad.

Cabe aquí subrayar que no es la postura sobre lo que hablan los estudios de lo que debemos hacer, sino sobre "mi" postura ante la realidad. Esto implica ser capaz de reconocer que me estoy preguntando algo, que esa pregunta sale de mi propia curiosidad, y que esa curiosidad no tiene por qué coincidir con el profesorado universitario y mucho menos con los autores o autoras que he leído. Ciertamente, esta situación es muy compleja, y este ensayo no trata de explicarla y mucho menos agotarla.

Si la curiosidad "propia" no es el punto de partida de una investigación, no podemos pretender que el estudiantado se apropie y "enamore" de su investigación, que reconozcan en ella una relación íntima que le permita verse como sujeto que decide en interacción con los sujetos investigados, y que puedan definir conjuntamente cuál es el camino que debe seguir la propuesta.

Reconocer la propia curiosidad que las preguntas iniciales del proceso investigativo provocan y que, además, son una construcción que surge de su vivencia, cuestionamientos y necesidades permite fortalecer su identidad futura como profesional, y que puede construir y sostener una "tesis" sobre la realidad que estudia. Al respecto, Freire y Faundez (2013) apuntan que "durante el proceso de buscar informaciones que ayuden a responder a estas preguntas, otras preguntas fundamentales emergen en la constitución de un cuerpo coherente, lógico, riguroso, que debe ser la tesis" (p. 75).

\section{Las propuestas de los cursos de investigación: Aprender haciendo}

A partir de la reflexión antes descrita, la CTFG se da a la tarea de vincular directamente el aprender a hacer, el ser y el saber desde un conocimiento praxeológico, profundamente relacionado con el quehacer pedagógico que el estudiantado desarrolla en el contexto elegido para su trabajo final de graduación.

De ahí, entonces, que los cursos de investigación en el nivel de licenciatura se plantean para que el conocimiento que se ofrece parta del proceso de investigación y de las necesidades que esta demanda. Al desarrollar estos procesos de forma paralela, el estudiantado universitario encuentra un sentido y un propósito en el reconocimiento de su postura epistemológica, ontológica, metodológica y conceptual, a partir de la organización de una experiencia de conocimiento que surge desde la pregunta de investigación. 
http://doi.org/10.15359/ree.24-2.24

ROR: https://ror.org/01t466c14 Universidad Nacional, Costa Rica

http://www.una.ac.cr/educare

educare@una.cr

La experiencia en la práctica universitaria lleva a concluir que, para promover un aprendizaje significativo a partir de la experiencia en investigación, es necesario que la persona que desarrolla estos procesos los construya en el "hacer" y formulando nuevas formas de acercamiento a la realidad, desde una posición democrática y humanizada del objeto-sujeto de estudio.

Por tanto, la apropiación teórica no se convierte en el marco de actuación que define un trabajo que realiza el estudiantado, sino más bien en una necesidad de profundizar en la comprensión del problema de investigación desde el sentido y la mirada que otros seres han construido sobre la cuestión, desde otras realidades y dimensiones de la práctica pedagógica. Además, dicha construcción se realiza desde los saberes que el estudiantado ha logrado construir a lo largo de su formación universitaria, así como de los nuevos retos que plantea el abordaje diverso de una problemática específica que requiere de una respuesta crítica, ajustada, pertinente y contextualizada.

En consecuencia, pensamos que esta forma de proceder es una manera diferente de hacer investigación, en la que no necesariamente se cumple con una serie de conocimientos técnicos, pero sí con la actitud aprendiente para iniciar la experiencia de trabajo investigativo. Se busca que la población estudiantil tenga la posibilidad de acercarse a una experiencia orgánica y vital, y no responder únicamente al cumplimiento obligatorio de un requisito para obtener su certificación de grado.

El aprender en la experiencia y de esta también abre las posibilidades a mayor diversidad y complejidad en la adquisición de las herramientas de investigación, enriqueciendo con ello una posición paradigmática en coherencia con los diversos contextos y realidades educativas. Las percepciones y comprensiones de los sujetos actuantes en la investigación se nutren entre sí, y la necesidad de plantear rutas metodológicas innovadoras y diferentes casi que se convierte en un requisito. Para tales efectos, se necesitará indagar más y profundizar más en la investigación cualitativa y las diferentes formas que se han desarrollado para el logro de procesos creativos, fundamentados en los criterios de calidad de la investigación que se desarrollan desde una visión que no es medible ni generalizable.

En resumen, la propuesta es que, desde los cursos de investigación, se lleve de manera paralela el proceso de investigación; esto, por cuanto se considera que la enseñanza de la investigación debe ser algo orgánico en el proceso de formación docente, que promueva la capacidad de cuestionar, preguntar y proponer. Además, una propuesta de esta índole debe partir de la revisión y profundización del posicionamiento teórico y epistemológico del estudiantado de licenciatura desde su rama profesional, de manera que el trabajo final de graduación contribuya a fortalecer su postura como pedagogo o pedagoga frente a la realidad educativa en que le corresponda trabajar. 


\section{El futuro pedagogo: Sujeto de su quehacer y protagonista de su historia}

La mayoría de las veces tenemos un discurso teórico que nos cuesta llevar a la práctica, condición que trasladamos a las aulas universitarias. Tal es el caso cuando hablamos de dar la oportunidad a los niños y niñas de ser sujetos de su proceso de aprendizaje, pero cuánto cuesta realizarlo en el aula universitaria. Este es otro de los retos que sustenta esta propuesta de cambio: ¿Cómo recuperar el sujeto en la investigación, siendo que ese papel debe vivirlo el estudiantado dentro de su propia propuesta de investigación? Es un reto, pues la propuesta educativa siempre lo ha ubicado, como estudiante, en el papel receptor, y a docentes en el de transmisión.

Cuando el estudiantado se egresaba de la licenciatura, esta calidad era débil dentro de su perfil profesional, sobre todo cuando se trata de producción de nuevo conocimiento. Por esa razón, una de las dificultades que presentaba el estudiantado cuando se egresaba de la licenciatura, con un anteproyecto en su mano, era poder defenderlo, poder reconocer frente a los otros individuos lo que quería investigar. Esto porque aún no tenían una apropiación clara del problema de investigación para sustentarlo frente a su tutor o tutora que, la mayoría de las veces, era la primera vez que tenía contacto con la propuesta del estudiantado, lo que generaba inseguridad en ambas partes. Sobre todo, afectaba al estudiantado que, eventualmente, debía modificar su propuesta de investigación sin tener la claridad suficiente para poder defenderla.

De ahí que darle espacio y tiempo al estudiantado para apropiarse de su curiosidad, tomar conciencia de la manera particular que ve el mundo, podría generar una posición diferente en relación a cómo se asume el proceso de investigación, y que es a partir de la pregunta, de la capacidad humana innata de cuestionar lo que le rodea y sus interacciones, como puede generarse un empoderamiento en la autogestión del conocimiento a partir del proceso de investigación.

El momento crítico en que se puede gestionar y guiar ese empoderamiento es cuando el estudiantado universitario está en las aulas, al contar con acompañamiento por parte del profesorado. Lejos de ser interpretada dicha guía como una intervención directa de la persona académica en el trabajo del estudiantado, se considera que la mediación pedagógica en el proceso de investigación puede contribuir a generar en él más autonomía y complejidad en los planteamientos que se hacen al trabajo, con lo que logra una mayor apropiación de su propuesta.

Este seguimiento puede también propiciar el conocimiento de otras herramientas, destrezas y habilidades en la investigación, ya que el desarrollo del TFG se nutre en la necesidad de encontrar nuevas posibilidades de actuación en una realidad compleja y cambiante, y a partir de la experiencia académica y profesional del equipo que lo asesora, el estudiantado puede plantear diseños metodológicos flexibles y ajustados a la experiencia.

Este apoyo de la persona docente universitaria es indispensable porque, como ya lo hemos expuesto, venimos de una formación escolarizada que no promueve la independencia intelectual y la creatividad en el acto pedagógico; por tanto, la experiencia de los cursos ha 
llevado al hallazgo de que no hay madurez suficiente por parte del estudiantado para asumir el proceso de investigación y, por tanto, se hace indispensable desarrollar los mecanismos para que se dé un proceso de construcción que posibilite esa madurez, ante los retos que genera el iniciar un TFG fundamentado en la investigación como fuente de conocimiento.

El estudiantado logrará este empoderamiento a partir de elementos fundamentales en la formación que se gestiona desde los cursos de investigación: la apropiación del objeto de investigación, la creatividad, el conocimiento de sus propias destrezas profesionales y el aporte que su TFG puede generar, no solo a la realidad en la que se desarrolla, sino también al enriquecimiento del campo de conocimiento de la profesión docente.

Este trabajo es posible si en la implementación de los planes de estudio de las carreras se plantea la investigación como una herramienta para la construcción del conocimiento; de igual manera, tareas propias como la apropiación teórica, observación, la sistematización, la elaboración de instrumentos y el análisis de información que se trabajan desde el ingreso a la carrera.

De no romper el hilo por alguna parte, se seguirán formando estudiantes que consumen una propuesta de sociedad en la que su puesto se reduce a consumir pasivamente. Con esto no solo nos referimos a los bienes materiales, sino también al conocimiento enlatado y cada vez más "relativo" que circula por el ciberespacio:"Vivimos un mundo donde las grandes preocupaciones no son conquistar el mundo sino crearse a [uno] mismo" (Gurdián-Fernández, 2010, p. 76).

Esta propuesta plantea grandes retos, pero se parte de que es un proceso permanente de construcción en el que el diálogo académico es la base que da sustento a la reflexión y construcción colectiva, la cual se propone revisar y reflexionar sobre los retos que propuestas como esta plantean. De ahí la necesidad de aprovechar que el estudiantado aún esté cursando la licenciatura para que no solo realice su anteproyecto, sino que también avance en su TFG, lo que permite que cuente con más acompañamiento. Ello posibilita lo siguiente:

- Crear más autonomía en el proceso de trabajo final de graduación: apropiación de su propuesta.

- Fortalecer la capacidad de asombro y creatividad mediante la pregunta.

- Contar con más apoyo docente, pues aún no hay madurez suficiente para asumir el proceso de manera independiente.

\section{Conclusiones}

La CTFG trabaja desde una posición epistemológica fundamentada en una pedagogía crítica, centrada en el aprendizaje, que se define a partir de la naturaleza de la construcción que se genera en la práctica pedagógica; asimismo, en cómo la persona que se forma en este 
campo de las ciencias sociales se apropia de dicho conocimiento, por una parte, mediante una investigación fundamentada en la capacidad de asombro y, por otra, en preguntarse acerca de las realidades. Un principio fundamental que podría consensuar este posicionamiento en la docencia universitaria es la necesidad de profesionales en pedagogía que no teman enfrentar los retos y desafíos que devienen de la complejidad de los contextos educativos del siglo XXI; en los que el estudiantado accede a la información y, al mismo tiempo, pueda generar sus propias herramientas de trabajo.

Esta concienciación acerca de la pregunta es el punto de partida para iniciar una práctica reflexiva que aspire, no al consumismo de teorías o metodologías que aseguran mejorar los procesos educativos, sino a la elaboración y diseño de propuestas pedagógicas contextualizadas y pertinentes, y a estudios investigativos que planteen una nueva relación sujeto/objeto de investigación, en una mirada más dialogante y solidaria. Se asume, entonces, que el quehacer universitario cobra sentido y significación, no solo para el estudiantado que realiza las investigaciones, sino también para las poblaciones que se espera beneficiar, en el marco de la reflexión que permite cuestionarse y crear caminos.

En síntesis, la propuesta de la investigación en el quehacer pedagógico de las carreras de la DEB a lo largo del proceso de formación docente tiene una intención desde una visión integral: construir a partir de la triada teoría- investigación-práctica; desde una visión crítica promover la investigación reflexión acción contextualizada y vivida; desde una visión constructiva, considerar la curiosidad propia, el diálogo y el posicionamiento epistemológico de cómo me aproximo a la realidad; $y$, finalmente, desde una visión humanista, al priorizar la trascendencia del sujeto-protagonista de las actividades humanas.

\section{Referencias}

Freire, P. (1979). Pedagogía del oprimido. Siglo XXI editores.

Freire, P. (1997). Pedagogía de la autonomía. Saberes necesarios para la práctica educativa. Siglo XXI editores.

Freire, P. y Faundez, A. (2013). Por una pedagogía de la pregunta. Crítica a una educación basada en respuestas a preguntas inexistentes. Siglo XXI editores.

Giménez, G. (1996). La identidad social o el retorno del sujeto en sociología. En L. Méndez (Comp.), Identidad, III Coloquio Paul Kirchhoff (pp. 183-205). México: UNAM-DGAPA.

Gurdián-Fernández, A. (2010). El paradigma cualitativo en la investigación socio-educativa. EUCR.

Ibáñez, J. (1994). El regreso del sujeto, la investigación social de segundo orden. Siglo XXI Editores. 
Jiménez, R. (2012). La investigación sobre coaching en formación del profesorado: Una revisión de estudios que impactan en la conciencia sobre la práctica docente. Profesorado Revista de curriculum y formación del profesorado, 16(1), 238-235. http://www.ugr.es/local/recfpro/ rev161COL2.pdf

Kemmis, S. (1999). Prólogo. En W Carr. (Autor). Una teoría para la educación. Hacia unainvestigación educativa crítica. Morata.

Mora Alvarado, M A. y Gómez Torres, J. R. (2017). Algunos fundamentos de investigación social. EUNA.

Patterson L. y Shannon, P. (2002). Reflexión, investigación y acción. En L. Patterson, C. Minnick Santa, K. G. Short y K. Smith (Eds.), Los maestros son investigadores. Reflexiones y Acciones (pp. 21-26). Trillas.

Programa Estado de la Nación. (2008). Estado de la Educación 2 (2a ed.). CONARE. https:// estadonacion.or.cr/informes/?current=2

Rillo, A. G., Pimentel, M. L., Arceo, M. E., Ocaña, H., García-Pérez, L. y Hernández-Monroy, D. (2011). Horizontes y estructura de la pregunta pedagógica. Revista Actualidades Investigativas en Educación, 11(1), 1-18. https://doi.org/10.15517/aie.v11i1.10173

Stenhouse. L. (2003). Investigación y desarrollo del curriculum. Morata. 